\title{
Recurrent AIPL1 c.487C >T truncating variant in Leber Congenital Amaurosis: Support of pathogenicity and regional implications
}

Mohammed O.E. Abdallah ${ }^{1 *}$ (melsiddieg@gmail.com), Mahmoud E. Koko ${ }^{1,2 *}$

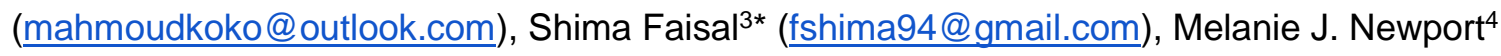

(M.J.Newport@bsms.ac.uk), Muntaser E. Ibrahim¹ (mibrahim@iend.org).

${ }^{1}$ Department of Molecular Biology, Institute of Endemic Diseases, University of Khartoum, Khartoum, Sudan.

${ }^{2}$ Department of Neurology and Epileptology, Hertie Institute for Clinical Brain Research, Tuebingen, Germany.

${ }^{3}$ Faculty of Pharmacy, University of Khartoum, Khartoum, Sudan.

${ }^{4}$ Wellcome Trust Brighton and Sussex Centre for Global Health Research, Brighton and Sussex Medical School, Brighton, UK.

${ }^{\star}$ Equal contribution.

Corresponding author: Prof. ME Ibrahim, Institute of Endemic Diseases, Department of Molecular Biology, University of Khartoum, 11111 Khartoum, Sudan. P.O. box 102. Email: mibrahim@iend.org. 


\section{Abstract:}

Background: Leber Congenital Amaurosis (LCA) is a clinically and genetically heterogeneous inherited retinal dystrophy characterized by early onset visual impairment caused by mutations in not less than 17 genes. AIPL1 mutations cause LCA type 4 , comprising approximately $7 \%$ of LCA worldwide. The importance of establishing a genetic diagnosis lies in the promise of gene therapy demonstrated in mouse models. Results: we genetically investigated a consanguineous Sudanese family with Leber Congenital Amaurosis. Eight members of the family were affected. Using whole exome sequencing in two siblings and their healthy mother, both inheritance-based and phenotype-based prioritization strategies converged to identify a truncating variant (rs62637009) in AIPL1, consistent with a diagnosis of LCA type 4. AIPL1 c.487C >T is an ultra-rare cause of LCA4 that was seen previously in homozygous state in a single Palestinian family. This recurrent variant seems to have a regional importance with a likely founder effect.

Conclusions: This report adds evidence to the pathogenicity of $A I P L 1 \mathrm{c} .487 \mathrm{C}>\mathrm{T}$ meriting its conclusive annotation as a recurrent pathogenic variant. This variant is particularly relevant to the middle-eastern and northeast African regions.

Keywords: Leber Congenital Amaurosis, LCA4, AIPL1 c.487C>T, Sudan, AR autosomal recessive.

\section{Introduction:}

Leber Congenital Amaurosis (LCA) is a clinically and genetically heterogeneous inherited retinal dystrophy characterized by early onset visual impairment and nystagmus. The diagnosis of LCA is usually clinical. Pathogenic variants in 17 genes are known to cause LCA and at least one other disease locus for LCA has been reported [1]. These genes encode a variety of proteins, including those involved in developmental and physiological pathways in the retina [2]. LCA type 4 (LCA4) is caused by AIPL1 gene located in chromosome 17p13.1. AIPL1 mutations cause approximately $7 \%$ of LCA worldwide. It was first identified in a Pakistani family with autosomal recessive amaurosis [3].

Mutations in AIPL1 are associated with a form of LCA that is characterized by maculopathy and a pigmentary retinopathy starting at a young age [4]. AIPL1 (aryl hydrocarbon receptor-interacting protein-like 1) which is expressed in rod and cone photoreceptors, has a critical role in cell viability and the assembly of the phototransduction protein, phosphodiesterase, in both rods and cones [5]. In mouse models of AIPL1 deficiency, rapid complete degeneration of both rods and cones is seen within 4 weeks of age [6,7]. Establishing a genetic diagnosis for LCA is of great importance as the prospects of gene therapy seems promising, especially for LCA caused by AIPL1 as demonstrated in mouse models [8].

Multiple genetic variations in AIPL1 has been reported to cause of LCA4. As of the end of September 2017, the ClinVar [9] database lists 10 pathogenic and likely pathogenic variants in AIPL1 including one truncation (p.(Trp278Ter)), two insertion-deletions 
(p.(Glu337Alafs) and p.(Al352_Pro355del)), and six missense (p.(Ala197Pro), p.(Ile206Asn), p.(Cys239Arg), p.(Gly262Ser), p.(Arg302Leu), p.(Pro376Ser)) variants, while more than 50 variants are reported in the HGMD database [10]. Here we report the first Sudanese family with autosomal recessive Leber Congenital Amaurosis resulting from AIPL1 c.487C>T (p.(Glu163Ter)) truncating variant (rs62637009) diagnosed using whole exome sequencing. To our knowledge, this is the first phenotype-genotype correlation of AIPL1 with LCA4 in the east African region, and the second report of $c .487 \mathrm{C}>\mathrm{T}$ worldwide that was originally seen in a Palestinian family [11].

\section{Methods:}

We genetically investigated two siblings with early onset visual impairment and a family history of a similar condition using whole exome sequencing. The first patient was a female aged twenty-four years, who was blind at time of investigation. The onset of visual impairment was noted since birth with severe loss of vision in early childhood (Acuity: Perception of Light). Photo-attraction was present (staring at light) as well as retinal pigmentation. She developed keratoconus and cataract resulting in complete blindness. Her younger brother (six years old) had a similar clinical presentation (acuity: Perception of Light). The family pedigree showed another affected sibling along with five related patients with similar phenotypes. Figure (1) shows the family pedigree.

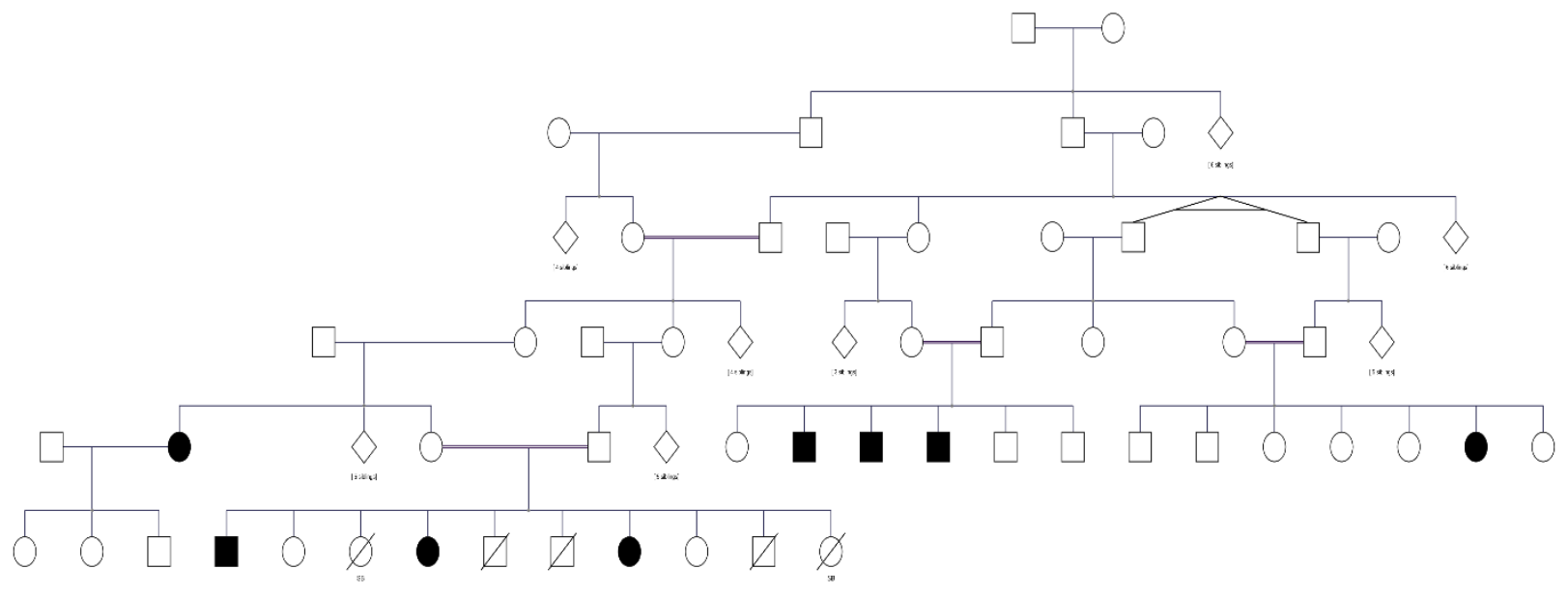

Figure 1 the pedigree of consanguineous multigenerational family with Leber congenital amaurosis.

Whole exome sequencing: Whole exome sequencing was performed on two affected probands and their mother. Paired-end sequencing was done on Illumina HiSeq platform (Illumina, CA, USA) using DNA extracted from peripheral blood samples. Agilent SureSelect enrichment kits were used (Agilent Technologies, CA, USA). Following alignment with BWA MEM [12], sorting and duplicates removal with Samtools [13], variants were called using Freebayes [14]. Variant calls were annotated using SnpEff v4.1 [15] and Ensembl VEP v86 [16]. The annotations included ExAC allele frequencies [17] and multiple pathogenicity predictions. 
Linkage-based Variant Prioritization: the consistency of the phenotype, its severity along with the consanguinity seen in the pedigree were highly suggestive of the inheritance of a rare or novel variant in a homozygous region. Gemini v0.18 [18] was used for identification of rare homozygous variants segregating in both patients. We performed homozygosity mapping using Homozygosity Mapper [19] and looked for homozygous runs shared by both patients. All regions with scores above $90 \%$ of the maximal exome wide homozygosity signal were prioritized. After the exclusion of the homozygous runs seen in the mother, we examined all the rare or novel variations in shared runs. Figure 2 Shows

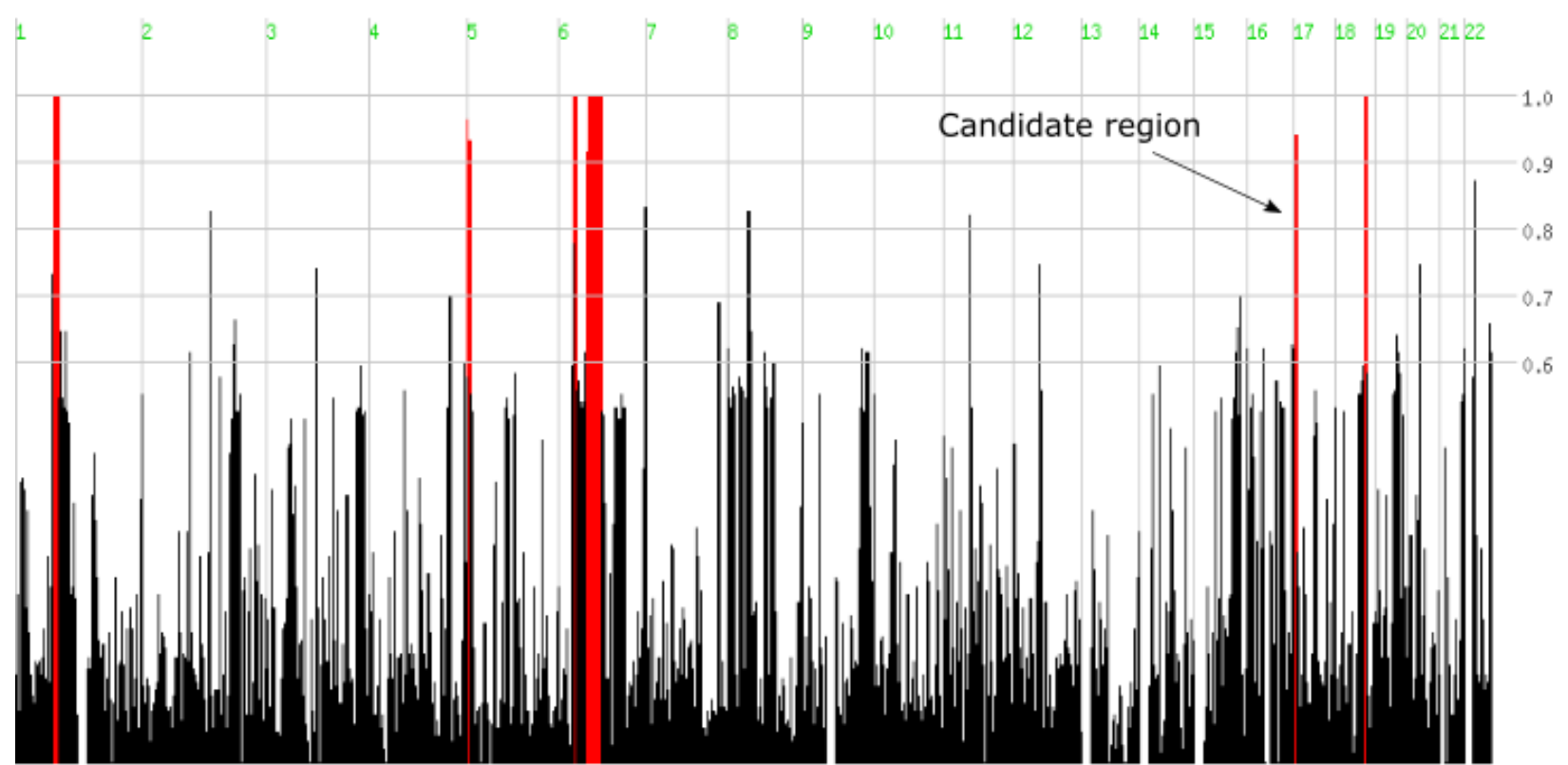

Figure 2 Illustrates runs of homozygosity (ROHs) identified by HomozygosityMapper

Phenotype-based Variant Prioritization: we obtained a list of genes linked to LCA in OMIM [20] database under the phenotypic series PS204000, which included seventeen genes. OpencgaR v1.3 (https://github.com/opencb/opencga/) was used to mine all variants overlapping this gene list from the VCF files. Scaled CADD[21] score cutoff of 20 was used for deleteriousness filtering. All variants exceeding this score were manually evaluated for their pathogenicity and relation to the phenotype. We also used Exomizer v8.0.1 [22] for automated variant prioritization (complete results and parameters for Exomizer analysis are available in supplementary materials file S1).

\section{Results}

Inheritance-based variant prioritization: The highest shared homozygosity signals were seen on chromosomes 5,6 (two runs of homozygosity), 17, and 18 with a length of $0.54 \mathrm{Mb}, 9.4 \mathrm{Mb}, 15.3 \mathrm{Mb}, 0.6 \mathrm{Mb}$, and $1.6 \mathrm{Mb}$, respectively. We excluded a region in 
chromosome 1 that was similarly homozygous in the mother. In total, only four rare variants were seen in these regions that fit a recessive inheritance pattern. The variant rs62637009 was the only pathogenic variant (stop gain). This truncating variant in AIPL1 (NM_014336.4:C.487C>T) was in a homozygous state in the patients and heterozygous carrier state in their mother with coverage exceeding $20 \mathrm{x}$ in all three samples. It is predicted to introduce an early stop codon (NP_055151.3:p.(Gln163*))( Figure (3). The remaining three variants (rs759751696, rs2089318, rs144622175) were all predicted to have a non-coding modifier effect. It is worth mentioning that the length of the homozygous run did not indicate the position of the causative variant.

Phenotype-based variant prioritization: 92 variants in LCA genes were detected, including four variants that followed an AR pattern of inheritance (rs62637009, rs2297129, rs2297128, and rs2274736). Only rs62637009 had a deleterious scaled CADD score above 20 (36). Interestingly, rs62637009 was also the top hit in Exomizer results, with a score of 0.818 , a phenotype score of 0.584 , and a variant score of 0.950 Clinical Interpretation of rs62637009 according to ACMG/AMP 2015 guidelines [23] Using ACMG wlntervar [24] this variant was classified as pathogenic on the basis of the following evidence codes: PVS1, PS1, PM2, and PP3 (null variant (nonsense), Same amino acid change as a previously established pathogenic variant regardless of nucleotide change, Absent from controls (or at extremely low frequency if recessive) in Exome Sequencing Project, 1000 Genomes Project, or Exome Aggregation Consortium, multiple lines of computational evidence support a deleterious effect on the gene or gene product)

\section{Discussion and conclusions:}

Identification of rare disease variants is critical for the accurate diagnosis, counseling and any future gene-specific therapeutic interventions. This presents an everyday challenge for clinicians and geneticists. Traditional sequencing of a limited number of genes is a strategy that fails to cope with the genetic heterogeneity seen in many disorders. Using population-optimized microarrays is yet another proposed solution that will likely struggle to accommodate the expanding number of known and novel variants with different frequencies that probably relate to each population's structure and evolutionary history. As well, when assessing an individual patient, the answer to the question 'which population is representative of each patient' might not be as clear as we expect.

Exome sequencing provides an exceptional opportunity to identify ultra-rare and novel variants in single families. However, the challenge of variant prioritization in exome sequencing studies is a big hurdle. In this study we demonstrated that employing an integrative approach combining genotype-based strategies like homozygosity mapping (in consanguineous families) and phenotype-based approaches, e.g. through Exomizer has the potential to converge on the same causative variant. While homozygosity mapping continues to prove its worth in identifying recessive disease variants, phenotype-based strategies coupled with deleteriousness score filtering are suitable for many scenarios of Mendelian and non-Mendelian inheritance. 
We identified two patients who harbor a rare homozygous nonsense variant in AIPL1 that has been previously reported only once in homozygous state [25]. The patients in the previous report by Tan et al. [11] were of Palestinian origin, highlighting the importance of extremely rare, population-specific disease-causing variants. This variant was not detected in many large databases including ExAC [17] and GnomAD indication a possible founder effect. The phenotype observed in our patients matched the phenotype previously described for this type of LCA with an early onset loss of vision characterized by a very low visual acuity (light perception) that further deteriorates following the development of keratoconus and cataracts as well as pigmentary retinopathy, also characteristic of AIPL1 forms of LCA [26]. Assessing this variant against the ACMG guidelines [23] clearly pinpointed its clinical significance, adding further evidence to its pathogenicity.

This variant is located distal to the FKBP-type peptidylprolyl isomerase domain and before the TPR domain (formed of Tetratricopeptide repeat-containing domain, Tetratricopeptide-like helical domain and a Tetratricopeptide repeat motif) and Cterminus Proline-rich domain. TPR domain mediates protein-protein interactions and the assembly of multiprotein complexes [27] . Variants affecting the TPR domain of AIPL1 are thought to disrupt its interaction with $\mathrm{Hsp90}$ and $\mathrm{Hsp} 70$ [28] preventing the formation of a retina-specific chaperone heterocomplex facilitating retinal protein maturation[28]. This variant results in a short truncated protein with loss of terminal domains, and is most likely eliminated by Nonsense-mediated decay (NMD), although this requires experimental validation. However, early null variants typically cause NMD with a subsequent protein deficiency. Figure 3 shows a lollipop plot of AIPL1 gene variants

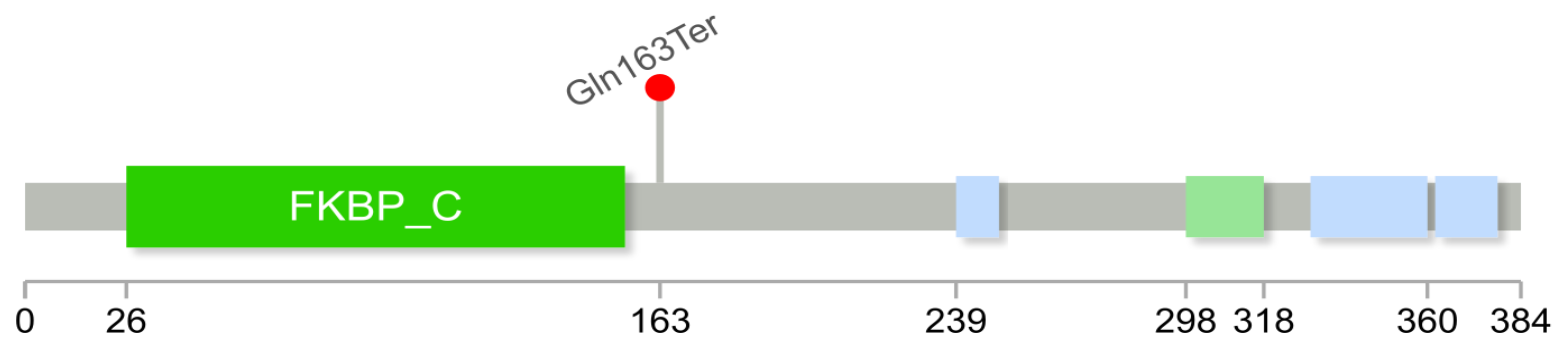

Low complexity region (Pfam/SEG)

FKBP-type peptidyl-prolyl cis-trans isomerase

Coiled-coil motif (Pfam/ncoils)

Figure 3 a lollipop plot that illustrates the impact of rs62637009 on the AIPL1 protein

In summary, there is enough evidence to implicate NM_014336.4(AIPL1):C.487C >T as a pathogenic ultra-rare truncation causing Leber Congenital Amaurosis with rapid and severe progression. This variant also seems to have a regional significance with a possible founder effect. 


\section{List of Abbreviations:}

Variant, NMD, LCA

\section{Declarations:}

Ethical approval and consent for publication: was obtained from the institutional ethical committee of the Institute of Endemic Diseases, University of Khartoum. Informed consent was obtained from adult participants and the parents of participating children for the genetic analysis, the publication of this report and all accompanying materials. All investigated individuals agreed to the publication of this report including their anonymous clinical data, family pedigree and genetic results.

Availability of data and materials: Supplementary file (I) is Exomizer results file. The raw exome datasets analyzed during the current study are not publicly available to abide to the confidentiality requested by the participants.

Competing Interests: the authors declare no conflicts of interests:

Funding: This work has been supported by the Research Development Fund, Wellcome Trust Brighton and Sussex Centre for Global Health Research grant no 100715/Z/12/Z .

Authors' Contribution: SF, MI and MN conceived and designed the study. SF obtained the data and processed the blood samples. MI and MN facilitated the exome sequencing. MA and MK analyzed the exome data and interpreted the results. MK and MA wrote the manuscript with critical revisions from SF, MI and MN. All authors read the manuscript and approved its final version.

\section{Acknowledgments:}

Not applicable.

\section{References:}

1. Weleber RG, Francis PJ, Trzupek KM, Beattie C: Leber Congenital Amaurosis. 1993.

2. den Hollander Al, Roepman R, Koenekoop RK, Cremers FPM: Leber congenital amaurosis: Genes, proteins and disease mechanisms. Prog Retin Eye Res 2008, 27:391-419.

3. Sohoki, Browne, Sullivan, Blackshaw, Cepko, Payne, Bhattacharya, Khaliq, Mehdi, Birch, Harrison, Elder, Heckenlively, Daiger: Mutations in a new photoreceptor-pineal gene on 17p cause leber congenital amaurosis. Nat gen 2000;24:79-83. Am J Ophthalmol 2000, 129:8345.

4. Dharmaraj S, Leroy BP, Sohocki MM, Koenekoop RK, Perrault I, Anwar K, Khaliq S, Devi RS, Birch DG, De Pool E, Izquierdo N, Van Maldergem L, Ismail M, Payne AM, Holder GE, Bhattacharya SS, Bird AC, Kaplan J, Maumenee IH: The Phenotype of Leber Congenital Amaurosis in Patients With AIPL1 Mutations. Arch Ophthalmol 2004, 122:1029.

5. Kirschman LT, Kolandaivelu S, Frederick JM, Dang L, Goldberg AFX, Baehr W, Ramamurthy 
V: The Leber congenital amaurosis protein, AIPL1, is needed for the viability and functioning of cone photoreceptor cells. Hum Mol Genet 2010, 19:1076-87.

6. Dyer MA, Donovan SL, Zhang J, Gray J, Ortiz A, Tenney R, Kong J, Allikmets R, Sohocki MM: Retinal degeneration in Aipl1-deficient mice: a new genetic model of Leber congenital amaurosis. Brain Res Mol Brain Res 2004, 132:208-20.

7. Ramamurthy V, Niemi GA, Reh TA, Hurley JB: Leber congenital amaurosis linked to AIPL1: a mouse model reveals destabilization of cGMP phosphodiesterase. Proc Natl Acad Sci U S A 2004, 101:13897-902.

8. Tan MH, Smith AJ, Pawlyk B, Xu X, Liu X, Bainbridge JB, Basche M, Mclntosh J, Tran HV, Nathwani A, Li T, Ali RR: Gene therapy for retinitis pigmentosa and Leber congenital amaurosis caused by defects in AIPL1: effective rescue of mouse models of partial and complete Aipl1 deficiency using AAV2/2 and AAV2/8 vectors. Hum Mol Genet 2009, 18:2099-114.

9. Landrum MJ, Lee JM, Benson M, Brown G, Chao C, Chitipiralla S, Gu B, Hart J, Hoffman D, Hoover J, Jang W, Katz K, Ovetsky M, Riley G, Sethi A, Tully R, Villamarin-Salomon R, Rubinstein W, Maglott DR: ClinVar: Public archive of interpretations of clinically relevant variants. Nucleic Acids Res 2016, 44:D862-D868.

10. Stenson PD, Ball E V., Mort M, Phillips AD, Shaw K, Cooper DN: The Human Gene Mutation Database (HGMD) and Its Exploitation in the Fields of Personalized Genomics and Molecular Evolution. In Curr Protoc Bioinforma. Volume Chapter 1. Hoboken, NJ, USA: John Wiley \& Sons, Inc.; 2012:Unit1.13.

11. Tan MH, Mackay DS, Cowing J, Tran HV, Smith AJ, Genevieve A, Dev-borman A, Henderson $\mathrm{RH}$, Moradi P, Russell-eggitt I, Maclaren RE, Robson AG, Cheetham ME, Thompson DA, Andrew R, Michaelides M, Ali RR, Moore AT: Leber Congenital Amaurosis Associated with AIPL1: Challenges in Ascribing Disease Causation, Clinical Findings , and Implications for Gene Therapy. 2012, 7.

12. Li H: Aligning sequence reads, clone sequences and assembly contigs with BWA-MEM. 2013.

13. Li H, Handsaker B, Wysoker A, Fennell T, Ruan J, Homer N, Marth G, Abecasis G, Durbin R: The Sequence Alignment/Map format and SAMtools. Bioinformatics 2009, 25:2078-2079.

14. Garrison E, Marth G: Haplotype-based variant detection from short-read sequencing. 2012:1-9.

15. Cingolani P, Platts A, Wang LL, Coon M, Nguyen T, Wang L, Land SJ, Lu X, Ruden DM: A program for annotating and predicting the effects of single nucleotide polymorphisms, SnpEff: SNPs in the genome of Drosophila melanogaster strain w1118; iso-2; iso-3. Fly (Austin) 2012, 6:80-92.

16. McLaren W, Gil L, Hunt SE, Riat HS, Ritchie GRS, Thormann A, Flicek P, Cunningham F: The Ensembl Variant Effect Predictor. Genome Biol 2016, 17:122.

17. Lek M, Karczewski KJ, Eric V, Hill AJ, Cummings BB, Tukiainen T, Donnell-luria AHO, Ware JS, Tiao G, Tusie-luna MT, Weisburd B, Won H: Analysis of protein-coding genetic variation in 60,706 humans. Nat Publ Gr 2016, 536:285-291.

18. Paila U, Chapman B a, Kirchner R, Quinlan AR: GEMINI: integrative exploration of genetic variation and genome annotations. PLoS Comput Biol 2013, 9:e1003153.

19. Seelow D, Schuelke M, Hildebrandt F, Nürnberg P: HomozygosityMapper--an interactive approach to homozygosity mapping. Nucleic Acids Res 2009, 37(Web Server issue):W593-9. 20. Hamosh A, Scott AF, Amberger JS, Bocchini C a., McKusick V a.: Online Mendelian Inheritance in Man (OMIM), a knowledgebase of human genes and genetic disorders. Nucleic Acids Res 2005, 33(November 2014):789-798.

21. Kircher M, Witten DM, Jain P, O'roak BJ, Cooper GM, Shendure J: A general framework for estimating the relative pathogenicity of human genetic variants. Nat Genet 2014, 46:310315. 
22. Smedley D, Jacobsen JOB, Jäger M, Köhler S, Holtgrewe M, Schubach M, Siragusa E, Zemojtel T, Buske OJ, Washington NL, Bone WP, Haendel MA, Robinson PN: Next-generation diagnostics and disease-gene discovery with the Exomiser. Nat Protoc 2015, 10:2004-2015. 23. Richards S, Aziz N, Bale S, Bick D, Das S: ACMG Standards and Guidelines Standards and guidelines for the interpretation of sequence variants: a joint consensus recommendation of the American College of Medical Genetics and Genomics and the Association for Molecular Pathology. 2015(January):1-20.

24. Li Q, Wang K: InterVar: Clinical Interpretation of Genetic Variants by the 2015 ACMGAMP Guidelines. Am J Hum Genet 2017, 100:267-280.

25. Tan MH, Mackay DS, Cowing J, Tran HV, Smith AJ, Wright GA, Dev-Borman A, Henderson $\mathrm{RH}$, Moradi P, Russell-Eggitt I, MacLaren RE, Robson AG, Cheetham ME, Thompson DA, Webster AR, Michaelides M, Ali RR, Moore AT: Leber congenital amaurosis associated with AIPL1: challenges in ascribing disease causation, clinical findings, and implications for gene therapy. PLoS One 2012, 7:e32330.

26. Genetics OM: The Phenotype of Leber Congenital Amaurosis in Patients With. 2016, 122(July 2004).

27. D'Andrea LD, Regan L: TPR proteins: the versatile helix. Trends Biochem Sci 2003, 28:655-62.

28. Hidalgo-de-Quintana J, Evans RJ, Cheetham ME, van der Spuy J: The Leber congenital amaurosis protein AIPL1 functions as part of a chaperone heterocomplex. Invest Ophthalmol Vis Sci 2008, 49:2878-87.

\section{Supplementary Material}

File S1 Exomizer results and analysis paramters 